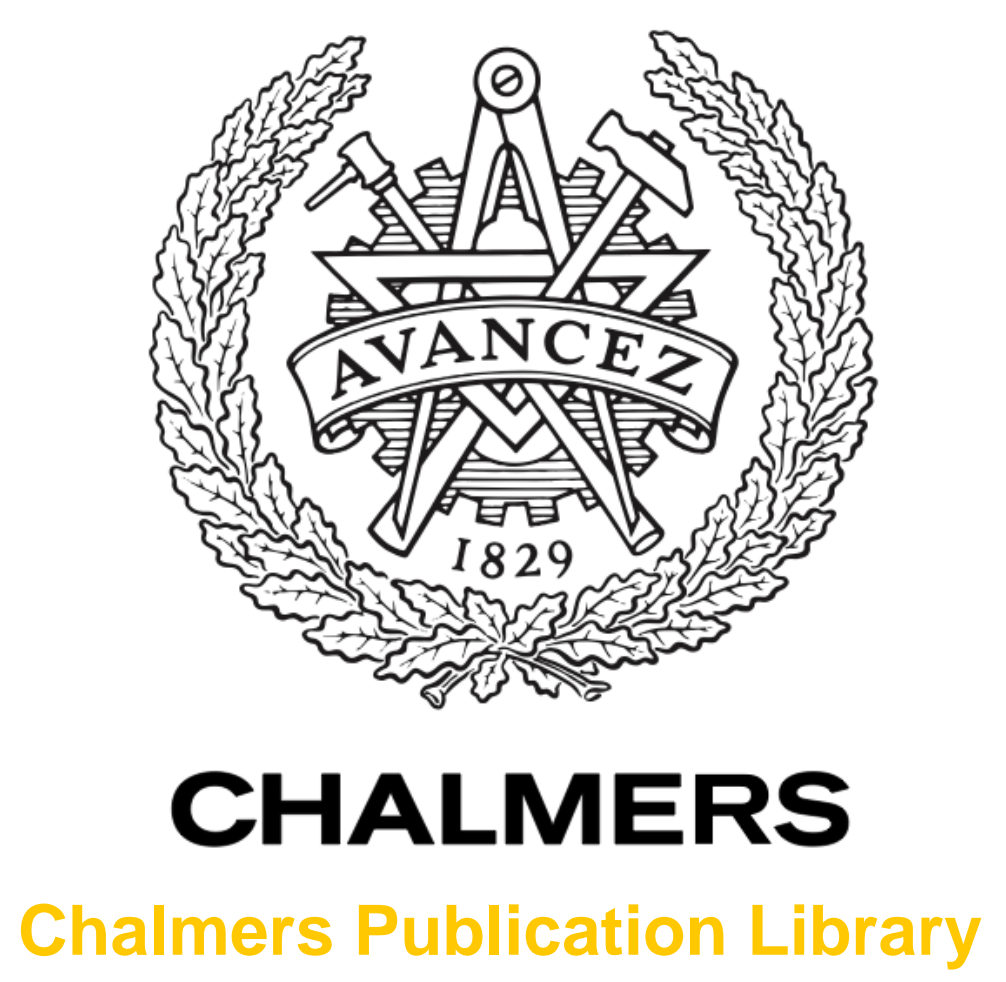

\title{
Stroke detection and diagnosis with a microwave helmet
}

This document has been downloaded from Chalmers Publication Library (CPL). It is the author's version of a work that was accepted for publication in:

Proceedings of 6th European Conference on Antennas and Propagation, EuCAP 2012. Prague, 26-30 March 2012

Citation for the published paper:

Fhager, A. ; Persson, M. (2012) "Stroke detection and diagnosis with a microwave helmet". Proceedings of 6th European Conference on Antennas and Propagation, EuCAP 2012.

Prague, 26-30 March 2012 pp. 1796-1798.

http://dx.doi.org/10.1109/EuCAP.2012.6206715

Downloaded from: http://publications.lib.chalmers.se/publication/160275

Notice: Changes introduced as a result of publishing processes such as copy-editing and formatting may not be reflected in this document. For a definitive version of this work, please refer to the published source. Please note that access to the published version might require a subscription.

Chalmers Publication Library (CPL) offers the possibility of retrieving research publications produced at Chalmers University of Technology. It covers all types of publications: articles, dissertations, licentiate theses, masters theses, conference papers, reports etc. Since 2006 it is the official tool for Chalmers official publication statistics. To ensure that Chalmers research results are disseminated as widely as possible, an Open Access Policy has been adopted.

The CPL service is administrated and maintained by Chalmers Library. 


\section{Stroke Detection and Diagnosis with a Microwave Helmet}

\author{
Andreas Fhager \\ Chalmers University of Technology \\ Department of Signals and Systems \\ SE-412 96 Göteborg \\ Sweden \\ Email: andreas.fhager@chalmers.se
}

\author{
Mikael Persson \\ Chalmers University of Technology \\ Department of Signals and Systems \\ SE-412 96, Göteborg \\ Sweden \\ Email: mikael.persson@chalmers.se
}

\begin{abstract}
In this paper we describe a microwave based mea- surement setup and a signal processing algorithm for stroke detection and diagnostics.
\end{abstract}

\section{INTRODUCTION}

In Sweden roughly 30000 people suffer a stroke each year and about $15-30 \%$ of them become permanently disabled, [1]. In the United States the corresponding number is approximately 700000 persons per year. Approximately $85 \%$ of all strokes are ischemic (blood clot induced) and 15\% hemorrhagic (bleeding) strokes. Stroke is placed third among reasons for acute death, and first among reasons for neurological dysfunction. Stroke is also resulting in most days of hospital nursing and therefore the most costly disease within the western world health care. The total social cost resulting from stroke in Sweden is estimated to EUR 1.2 billions per year. Among stroke survivors, $20 \%$ have serious remaining dysfunctions, [1]. A much larger proportion has less conspicuous dysfunctions which still seriously affect quality of life for the patient and relatives.

Early thrombolysis has proved very successful for ischemic stroke patients. If given to hemorrhagic stroke patients the treatment in itself could instead be lethal. The effect of the treatment is better the sooner it is given and it cannot be given later than 3 hours since start of the symptoms, as the tissue damage at this time result in a high risk for bleeding, [2]. Today computer tomography (CT) and sometimes magnetic resonance imaging (MRI) are used to diagnose the ischemic from the hemorrhagic strokes. The examinations in these facilities are however time consuming and together with transportation and other procedures in the chain of care the result is that only 2-3\% of the ischemic stroke patients receives the thrombolytic therapy.

Microwave technology is a strong and novel candidate to fulfill the needed gap of a diagnostic method in the care of acute stroke patients. It has high sensitivity to differentiate between blood and brain tissue due to the high difference in permittivity, [3]. It is also a technology with components that can be made compact and thus be implemented earlier in the health care chain, in emergency rooms and in ambulances. The same microwave technology has also attracted extensive interest in the research community as a novel method for breast cancer detection as well as several other applications that are under investigation.

Previously we have made a numerical feasibility study of the possibility to detect a blood clot inside the brain, [4]. We have also made an experimental study using tissue like phantom material. Both these studies have shown that the proposed microwave technique could be a viable method to detect bleedings. Currently we are performing a clinical study on 20 stroke patients of which 10 have a bleeding stroke and 10 a blood clot. For comparison we also make measurements on healthy volunteers. The study is made in collaboration with the Sahlgrenska University Hospital and the company Medfield Diagnostics AB. All clinical trials are approved by an Ethics Committee and the Medical Products Agency in Sweden. The measurements on the patients are made after they have gone through the normal diagnostic procedures and received treatment in order not to put the patient under any additional risk by interfering during the acute phase.

\section{MEASUREMENT SETUP:}

The measurement strategy in this application is similar to what is traditionally used in microwave tomography applications. A number of transmitting and receiving antennas are mounted in a configuration surrounding the target object under investigation, in this case the skull. Data is measured using every possible combination of antennas as transmitter and 
receiver. For the measurements we use 10 triangular patch antennas with a V-shaped slot and a shortcircuiting wall between the patch and the ground plane. The 10 antennas can be seen mounted inside a normal bicycle helmet in Fig. 1. It is also necessary to use

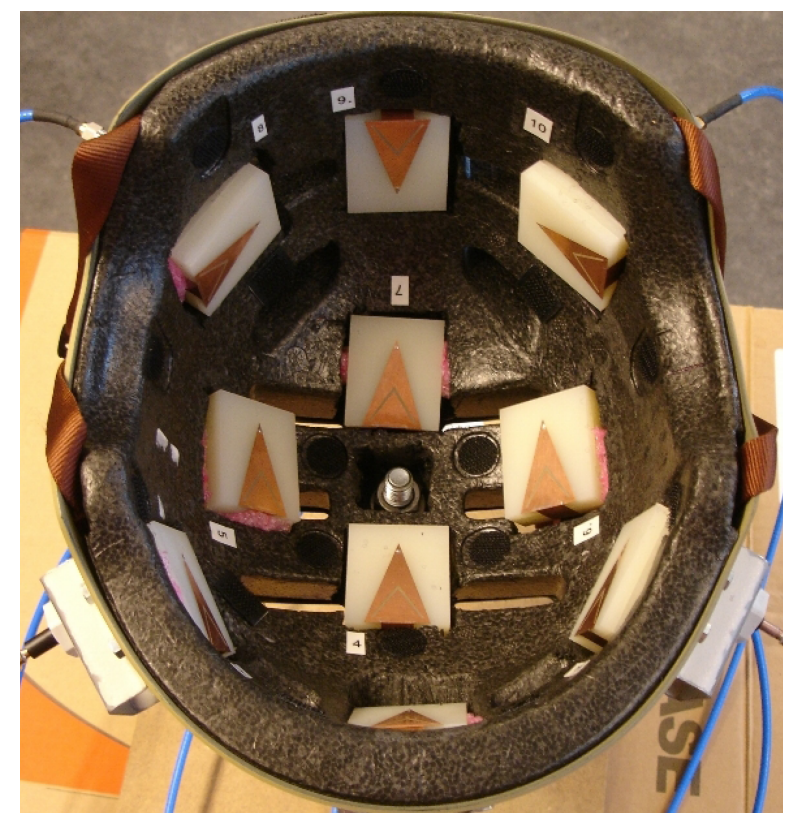

Fig. 1. A photo of the microwave helmet. The patch antennas can be seen mounted inside a bicycle helmet.

a matching medium between the antennas and the skull to couple the signal into the skull. A gap of air between the antenna and the skull could be disastrous to the development of a successful stroke detection application. A significant reflection would occur in the interface between the skin and the air due to the large difference in impedances. In breast cancer microwave tomography it is common to immerse the antennas and also the breast in some matching liquid before the measurements are made. For obvious reasons it is not a viable solution to submerge the skull of the stroke patient into a matching medium before the measurements. In this study we instead used plastic bags filled with normal water as matching liquid. The water bags were attached inside the helmet, covering the antennas. A system for adjusting the amount of water in the bags was used in order to adjust the fitting of the helmet on the patient, to accommodate different sizes of the skull and to make sure that the space between the antennas and the skull was completely filled with the matching liquid. The water bags are however not shown in the photo in Fig. 1.

The antennas are designed to have the desired property in the vicinity of the lossy media in form of the skull. However the characteristics of the anten- nas are sensitive and changing with the surrounding media. They have a resonance frequency of about 1.3 $\mathrm{GHz}$ in the actual measurement situation. Data in the frequency range $0.1 \mathrm{GHz}$ to $3.0 \mathrm{GHz}$ is collected using a network analyzer.

\section{SIGNAL PROCESSING:}

In order to interpret the measured data and to make the diagnosis it is necessary to use some signal processing algorithm. We are currently investigating two different types of algorithms. One is a full dielectric reconstruction using inverse algorithms from microwave tomography, citeFhager2006. The other is a classifier based on calculated subspace distances in the measured data and a training set of data from patients with known diagnosis, [6].

In the full reconstruction the dielectric parameters inside the antenna array are determined from the measured scattering data. In principle all the different methodology currently being researched for image reconstruction in microwave tomography applications can be utilized. The reconstruction problem is nonlinear and it means that an iterative optimization procedure is necessary. As a measure of the convergence we use an objective functional containing the difference between the measured and corresponding numerically simulated data. We use an approach where we compute gradients from a FDTD simulation of the antenna array and an adjoint electromagnetic problem. The gradients are used with a conjugate gradient algorithm and a line search to find the optimal step length that minimizes the functional. Currently we are working on the the FDTD modeling as this algorithm puts high demands on accurate modeling of the antenna array.

In the classifier algorithm we attempt to identify and separate the hemorrhagic stroke patients from the healthy and the ischemic stroke patients. The classifier is based on the assumption that a measurement is a sample of a random variable with a certain mean value and covariance matrix. Further it is assumed that the distribution, i.e. the mean and variance, is dependent on whether the data originated from a patient with a bleeding or a clot. Classifiers are based on some metric measures to separate the different classes. The situation here is that we have data from a large number of healthy patients but relatively fewer patients with stroke and therefore we have found a subspace distance measure most useful. The subspace method is most suitable when the dimension of the data set is greater than the number of the available samples and the number of samples of one class is larger than other class. To separate bleeding patients from the healthy ones and the patients with a clot 
it is necessary to use training data to determine a threshold level in the distance measure that separates the different classes. Once the threshold level has been determined a patient can be diagnosed. Experimental results from a phantom study shows promising results when it comes to separating the bleeding patients from the non-bleeding. We have also seen that the subspace distance potentially is a viable measure to determine the size of the bleeding. An improved version of the helmet is now in a second round of clincial studies.

\section{CONCLusions:}

We have built an experimental prototype for microwave diagnostics of stroke patients. The aim is to separate bleeding strokes from healthy patients and patients with a blood clot. 10 antennas are mounted inside a helmet and worn by the patient during the examination. We are primarily investigating a signal processing algorithm which is a statistical classifier algorithm. The results have been promising so far and the an improved version of the helmet is now in a second round of clinical evaluations. Results will be presented at the conference.

\section{REFERENCES}

[1] B. Norrving, "Förekomst i befolkningen," Vårdalinstitutets Tematiska rum : Stroke vård, omsorg och rehabilitering, 2007, (Access at: www.vardalinstitutet.net).

[2] The National Board of Health and Welfare of Sweden, "National guidelines for stroke care 2005", Sweden, 2006, (Access at: www. sos.se).

[3] S. Gabriel, R. W. Lau and C. Gabriel, "The dielectric properties of biological tissues: II. Measurements in the frequency range $10 \mathrm{~Hz}$ to $20 \mathrm{GHz}$,' Phys. Med. Biol., vol. 41, pp. 2251-2269, 1996.

[4] H. Trefna, M. Persson, "Antenna array design for brain monitoring", Antennas and Propagation Society International Symposium, (AP-S 2008), San Diego, CA, 5-11 July 2008.

[5] A. Fhager, P. Hashemzadeh and M. Persson, "Reconstruction quality and spectral content of an electromagnetic time-domain inversion algorithm," IEEE Trans. Biomed. Eng., vol. 53, no. 8, pp. 1594-1604, Aug. 2006.

[6] M.A. Khorshidi, T. McKelvey, M. Persson, H. Dobsicek Trefna, "Classification of Microwave Scattering Data based on a Subspace Distance with Application to Detection of Bleeding Stroke", The Third International Workshop on Computational Advances in Multi-Sensor Adaptive Processing (CAMSAP 2009), Aruba, Dutch Antilles, 13-16 December 2009. 